\title{
QUASI-CONFORMAL RIGIDITY OF NEGATIVELY CURVED THREE MANIFOLDS *
}

\author{
YONG $\mathrm{HOU}^{\dagger}$
}

\begin{abstract}
In this paper we study the rigidity of infinite volume 3-manifolds with sectional curvature $-b^{2} \leq K \leq-1$ and finitely generated fundamental group. In-particular, we generalize the Sullivan's quasi-conformal rigidity for finitely generated fundamental group with empty dissipative set to negative variable curvature 3-manifolds. We also generalize the rigidity of Hamenstädt or more recently Besson-Courtois-Gallot, to 3-manifolds with infinite volume and geometrically infinite fundamental group.
\end{abstract}

1. Introduction. Let $\widetilde{M}$ be a simply connected complete Riemannian manifold with sectional curvature $-b^{2} \leq K \leq-1$. Let $\operatorname{ISO}(\widetilde{M})$ denote the group of isometries of $\widetilde{M}$. Let $\Gamma$ be a non-elementary, torsion-free, discrete subgroup of $\operatorname{ISO}(\widetilde{M})$, and set $M:=\widetilde{M} / \Gamma$.

First we recall some terminologies that is required for the statement of the theorem. Let $S_{\infty}$ denote the boundary of $\widetilde{M}$. On $S_{\infty}$ one can define a metric in the following way. Let $v$ be a vector in the unit tangent bundle $S \widetilde{M}$. The geodesic $v(t)$ defines two points on $S_{\infty}$ given by $v(\infty)$ and $v(-\infty)$. Let $\pi_{t}$ be the projection of $S_{\infty} \backslash v(-\infty)$ along the geodesics which are asymptotic to $v(-\infty)$ to the horosphere which is tangent to $v(-\infty)$ and passing through $v(t)$. Let $\operatorname{dist}_{v, t}$ be the distance on the horosphere induced by restriction of the Riemannian distance, dist. On $S_{\infty} \backslash v(-\infty) \times S_{\infty} \backslash v(-\infty)$ define a function $\eta_{v}$ as $\eta_{v}(\xi, \zeta):=e^{-l_{v}(\xi, \zeta)}$ with $l_{v}(\xi, \zeta):=\sup \left\{t \mid \operatorname{dist}_{v, t}\left(\pi_{t}(\xi), \pi_{t}(\zeta)\right) \leq 1\right\}$. By our curvature assumption $-b^{2} \leq K \leq-1$, the function $\eta_{v}$ is a distance on $S_{\infty} \backslash v(-\infty)$, see [25].

Every element of $\gamma \in \Gamma$ has either exactly one or two fixed points in $S_{\infty}$, and $\gamma$ is called loxodromic if it has two fixed points [4]. The group $\Gamma$ is called purely loxodromic if all $\gamma \in \Gamma$ are loxodromic. The limit set of $\Gamma$ denoted by $\Lambda_{\Gamma}$ is the unique minimal closed $\Gamma$-invariant subset of $S_{\infty}$ [22]. If $\Gamma$ is purely loxodromic and $\Lambda_{\Gamma}=S_{\infty}$, then it can be either cocompact or $\widetilde{M} / \Gamma$ is geometrically infinite, hence $\Gamma$ has infinite co-volume. The convex hull $C H_{\Gamma}$ is the smallest convex set in $\widetilde{M} \cup S_{\infty}$ containing $\Lambda_{\Gamma}$. The group $\Gamma$ is called convex-cocompact if $C H_{\Gamma} / \Gamma$ is compact.

The critical exponent of $\Gamma$ is the unique positive number $D_{\Gamma}$ such that the Poincaré series of $\Gamma$ given by $\sum_{\gamma \in \Gamma} e^{-s \operatorname{dist}(x, \gamma x)}$ is divergent for $s<D_{\Gamma}$ and convergent for $s>D_{\Gamma}$. If the Poincaré series diverges at $s=D_{\Gamma}$ then $\Gamma$ is called divergent.

Let $f:\left(X, \rho_{X}\right) \longrightarrow\left(Y, \rho_{Y}\right)$ be a embedding between two topological metric spaces. Then $f$ is called quasi-conformal embedding [47] if there exists a constant $\kappa>0$ such that, for any $x \in X$ and $r>0$ there is $r_{f}(x, r)>0$ with

$$
f(X) \cap B^{\prime}\left(f(x), r_{f}(x, r)\right) \subset f(B(x, r)) \subset B^{\prime}\left(f(x), \kappa r_{f}(x, r)\right) .
$$

where $B$ and $B^{\prime}$ denotes a ball in $X$ and $Y$ respectively. When $f(X)=Y$ then $f$ is a quasi-conformal homeomorphism.

A torsion-free discrete subgroup $\Gamma$ of $\operatorname{ISO}(\widetilde{M})$ is called topologically tame if $\widetilde{M} / \Gamma$ is homeomorphic to the interior of a compact manifold-with-boundary.

\footnotetext{
${ }^{*}$ Received July 17, 2002; accepted for publication September 13, 2002.

tDepartment of Mathematics, University of Iowa, Iowa City, Iowa 52242, USA (yhou@math.uiowa.edu).
} 
THEOREM 1.1. Let $\Gamma^{\prime} \subset \operatorname{PSL}(2, \mathbb{C})$ be a topologically tame discrete group with $\Lambda_{\Gamma^{\prime}}=S^{2}$, and isomorphic $\chi: \Gamma^{\prime} \longrightarrow \Gamma$ to a convex-cocompact discrete subgroup $\Gamma$ of $\operatorname{ISO}(\widetilde{M})$ (here $\widetilde{M}$ is n-dimensional). Let $f: S^{2} \longrightarrow S_{\infty}$ be a quasi-conformal embedding which conjugate $\Gamma^{\prime}$ to $\Gamma$, i.e. $f \circ \gamma=\chi(\gamma) \circ f$, for $\gamma \in \Gamma^{\prime}$. Then $D_{\Gamma} \geq D_{\Gamma^{\prime}}$, and equality if and only if $\mathbb{H}^{3}$ embeds isometrically into $\widetilde{M}$ and the action of $\Gamma$ stabilizes the image.

To state our next theorem we need to introduce one additional terminology. We take $\widetilde{M}$ to be a 3 -manifold in the following.

Let $\mathfrak{M}_{\eta_{v}}^{\lambda}$ denote the $\lambda$-dimensional hausdorff measure on $\left(S_{\infty} \backslash v(-\infty), \eta_{v}\right)$. We say $\Gamma$ is hausdorff-conservative if there exists a constant $\alpha(v)>0$ such that $\alpha^{-1} r^{D_{\Gamma}} \leq \mathfrak{M}_{\eta_{v}}^{D_{\Gamma}}\left(B(\xi, r) \cap \Lambda_{\Gamma}\right) \leq \alpha r^{D_{\Gamma}}$ for any ball $B(\xi, r)$ of radius $r$ about $\xi \in \Lambda_{\Gamma}$ in $\left(S_{\infty} \backslash v(-\infty), \eta_{v}\right)$. From this definition, we note that if $\Gamma$ is a finitely generated torsion-free discrete subgroup of $\operatorname{PSL}(2, \mathbb{C})$ with $D_{\Gamma}=2$, then hausdorff-conservative implies conservative (classical definition, $\S 5$ ). Conversely, if $\Gamma$ is a topologically tame, conservative, discrete subgroup of $\operatorname{PSL}(2, \mathbb{C})$, then $\Gamma$ is hausdorff-conservative, see Proposition 5.2. We believe all finitely generated conservative discrete subgroup of $\operatorname{PSL}(2, \mathbb{C})$ are hausdorff-conservative, see Remark 5.3. For a convex-cocompact $\vec{M} / \Gamma$ with $-b^{2} \leq K \leq-1$, it follows from [12], $\Gamma$ is hausdorff-conservative. Now we are ready to state the theorem which generalizes Sullivan's quasi-conformal rigidity theorem.

THEOREM 1.2 (Main). Let $\Gamma$ be a topologically tame, purely loxodromic discrete subgroup of $\operatorname{ISO}(\widetilde{M})$ with $\Lambda_{\Gamma}=S_{\infty}$. Let $\Gamma^{\prime}$ be a topologically tame discrete subgroup of PSL $(2, \mathbb{C})$. Suppose $f: S_{\infty} \longrightarrow S^{2}$ is a quasi-conformal homeomorphism conjugate $\Gamma$ to $\Gamma^{\prime}$. Then $D_{\Gamma} \geq D_{\Gamma^{\prime}}$, and $\Gamma=\gamma \Gamma^{\prime} \gamma^{-1}$ with $\gamma \in \operatorname{PSL}(2, \mathbb{C})$ if and only if $D_{\Gamma}=D_{\Gamma^{\prime}}$ and $\Gamma$ is hausdorff-conservative.

COROllary 1.3. Let $M=\widetilde{M} / \Gamma$ be a complete topologically tame 3-manifold with $-b^{2} \leq K \leq-1, \Gamma$ purely loxodromic, and $\Lambda_{\Gamma}=S_{\infty}$. Let $h: M \longrightarrow N$ be a quasi-isometric homeomorphism to a hyperbolic manifold $N$. Then $M$ is isometric to $N$ if and only if $D_{\Gamma}=2$ and $\Gamma$ is hausdorff-conservative.

Let us point out that Theorem 1.2, generalizes known rigidity theorems in two directions for three dimensional manifolds.

First assume $M$ is hyperbolic $(b=1)$ but not necessarily geometrically finite. Since $M$ is topologically tame and $\Lambda_{\Gamma}=S^{2}$ we have $D_{\Gamma}=2$ by analytical tameness (see Proposition 3.3). Hence by Theorem 1.2, $M$ is quasi-conformal stable. This is a case of the Sullivan rigidity theorem for topologically tame $\Gamma$ with empty dissipative set. Next let us assume $M$ is compact with $-b^{2} \leq K \leq-1$. Then the critical exponent $D_{\Gamma}$ is equal to $h_{M}$ the topological entropy of $M$, and by [16], any homotopy equivalence between $M$ and a compact hyperbolic 3-manifold is induced by a homeomorphism. Therefore it follows from Corollary 1.3 we have: $M$ is isometric to a compact hyperbolic 3-manifold if and only if they are homotopically equivalent and $h_{M}=2$. This is the Hamenstädt's rigidity or more recently Besson-Courtois-Gallot theorem for 3-manifolds.

Note that it also follows from Theorem 1.2, the quasi-conformal version of the Hamenstädt's theorem for compact 3-manifold $M$ can be stated as:

COROLLARY 1.4. Let $\Gamma$ be a cocompact discrete subgroup of $\operatorname{ISO}(\widetilde{M})$. Let $\Gamma^{\prime} \subset \operatorname{PSL}(2, \mathbb{C})$ be a discrete group. Suppose $f: S_{\infty} \longrightarrow S^{2}$ is a quasi-conformal 
homeomorphism conjugate $\Gamma$ to $\Gamma^{\prime}$, Then $D_{\Gamma} \geq D_{\Gamma^{\prime}}$, and equality if and only if $\widetilde{M} / \Gamma$ is isometric to $\mathbb{H}^{3} / \Gamma^{\prime}$.

The proves of these theorems relies on our next result,

THEOREM 1.5. Let $M=\tilde{M} / \Gamma$ be a topologically tame 3 -manifold with $-b^{2} \leq$ $\mathcal{K} \leq-1$. Suppose that $\Gamma$ is purely loxodromic and that $\Lambda(\Gamma)=S_{\infty}$. Then $2 \leq D$ and $\Gamma$ is harmonically ergodic. If $D=2$ then $\Gamma$ is also divergent.

In section 2, we state some of the topological properties of negatively pinched 3-manifolds. In particular, we define geometrically infinite ends for negatively pinched 3-manifolds, and then state our theorem which describe the geometrical properties of this type of end, it is a crucial step in the proof of Theorem 1.5. Section 3 discusses measures on $S_{\infty}$ and the ergodicity of $\Gamma$ with respect to these measures. In section 4 , we give proofs of part I of the theorems. And section 5 is used to complete the proofs.

Acknowledgments: I am very grateful to Peter Shalen for his unwavering support and encouragement. I wish to thank Marc Culler, Dick Canary for their interest.

2. Topological Ends. Every isometry of $\tilde{M}$ can be extend to a Lipschitz map on $S_{\infty}:=\partial \tilde{M}$ [22]. For a torsion-free $\Gamma$, every element $\gamma \in \Gamma$ is one of the following types: (1) parabolic if it has exactly one fixed point in $\tilde{M} \cup S_{\infty}$ which lies in $S_{\infty}$; (2) loxodromic if it has exactly two distinct fixed points in $\tilde{M} \cup S_{\infty}$, both lying in $S_{\infty}$.

Denote by $\Lambda(\Gamma) \subset \partial \tilde{M}$ the limit set of $\Gamma$, which is the unique minimal closed $\Gamma$-invariant subset of $S_{\infty}$. Most of the important properties of the limit set in the constant curvature space continue to hold in the variable curvature space [15]. In particular: (i) $\Lambda(\Gamma)=\overline{\Gamma x} \cap S_{\infty}$; (ii) $\Lambda(\Gamma)$ is the closure of the set of fixed points of loxodromic elements of $\Gamma$; and (iii) $\Lambda(\Gamma)$ is a perfect subset of $\Gamma$. The set $\Omega(\Gamma):=$ $S_{\infty} \backslash \Lambda(\Gamma)$ is the region of discontinuity. The action of $\Gamma$ on $\tilde{M} \cup \Omega(\Gamma)$ is proper and discontinuous, see [15]. The manifold $M_{\Gamma}:=\tilde{M} \cup \Omega(\Gamma) / \Gamma$ with possibly nonempty boundary is traditionally called the Kleinian manifold. We also let $\Lambda_{c}(\Gamma)$ denote the conical limit set of $\Gamma$, i.e. $\xi \in \Lambda_{c}(\Gamma)$ if for some $x \in \tilde{M}$ (and hence for every $x$ ) there exist a sequence $\left(\gamma_{n}\right)$ of elements in $\Gamma$, a sequence $\left(t_{n}\right)$ of real numbers, and a real number $C>0$, such that $\gamma_{n} x \longrightarrow \xi$ and $\operatorname{dist}\left(c_{x}^{\xi}\left(t_{n}\right), \gamma_{n} x\right)<C$ where $c_{x}^{\xi}$ is the geodesic ray connecting $x$ and $\xi$. Equivalently, a point belongs to $\Lambda_{c}(\Gamma)$ if it belongs to infinitely many shadows cast by balls of some fixed radius centered at points of a fixed orbit of $\Gamma$. Note that $\Lambda_{c}(\Gamma)$ is a $\Gamma$-invariant subset of $\Lambda(\Gamma)$, hence a dense subset.

Proposition 2.1 (Margulis Lemma). There exists a number $\epsilon_{b}$ which only depend on the pinching constant $b$ of $M$, such that the group $\Gamma_{\epsilon}$ generated by elements in $\Gamma$ of length at most $\epsilon_{b}$ with respect to a fixed point in $M$ is almost nilpotent of rank at most 2. Then the number, $2 \epsilon_{b}$ is called the Margulis constant.

Note that, if $M$ is orientable and $\Gamma$ is torsion-free, then Margulis Lemma implies $\Gamma_{\epsilon_{b}}$ is abelian.

Let $\epsilon \leq \epsilon_{b}$ be given. Then $M$ may be written as the union of a thin part $M_{[0, \epsilon)}$ consisting of all points at which there is based a homotopically nontrivial loop of length $\leq \epsilon$ and a thick part $M_{[\epsilon, \infty)}=\overline{M-M_{[0, \epsilon)}}$. Note that $M_{[\epsilon, \infty)}$ is compact if 
$M$ is of finite volume. Also the thin part of $M$ is completely classified by the next proposition.

Proposition 2.2. Each connected component of $M_{[0, \epsilon)}$ is diffeomorphic to one of the following :

parabolic rank-1 cusp : $S^{1} \times \mathbb{R} \times[0, \infty)$.

parabolic rank-2 cusp : $T^{2} \times[0, \infty)$.

solid torus about the axis of a loxodromic $\gamma: D^{2} \times S^{1}$.

For simplicity we restrict to the case where $M$ has no cusps. It follows from the existence of a compact core $C(M)$ for $M$ [14], that $M$ has only finitely many ends [5]. In fact, each component of $\partial C(M)$ is the boundary of a neighborhood of an end of $M$, and this gives a bijective correspondence between ends of $M$ and components of $\partial C(M)$.

We define the simplicial ruled surfaces as follows. Let $S$ be a surface of positive genus and let $T_{P}$ be a triangulation defined with respect to a finite collection $P$ of points of $S$. This means that $T_{P}$ is a maximal collection of nonisotopic essential arcs with end points in $P$; these arcs are the edges of the triangulation, and the components of the complement in $S$ of the union of the edges are the faces. Let $f: S \longrightarrow M$ be a map which takes edges to geodesic arcs and faces to nondegenerate geodesic ruled triangles in $M$. The map $f$ induces a singular metric on $S$. If the total angle about each vertex of $S$ with respect to this metric is at least $2 \pi$, then the pair $(S, f)$ is called a simplicial ruled surface. It follows from the definition of the induced metric on $S$ that $f$ preserves lengths of paths and is therefore distance non-increasing. Any geodesic ruled triangle in $M$ has Gaussian curvature at most $-a^{2}$. This means that each 2-simplex of $S$ inherits a Riemannian metric of curvature at most $-a^{2}$. Since we have required the the total angle at each vertex to be at least $2 \pi$, by Gauss-Bonnet theorem the curvature of $S$ is negative in the induced metric.

Definition 2.3. An end $E$ is said to be a geometrically infinite if there exists a divergent sequence of geodesics, i.e: there exists a sequence of closed geodesics $\alpha_{k} \subset$ $M_{\epsilon}^{\circ}$, such that for any neighborhood $U$ of $E$, there exists some positive integer $N$ such that $\alpha_{k} \subset U$ for all $k>N$. If in addition for some surface $S_{E}$ we have that $U$ is homeomorphic to $S_{E} \times[0, \infty)$, and there exists a sequence of simplicial ruled surfaces $: S_{E} \stackrel{f_{l}}{\longrightarrow} U$ such that $f_{l}\left(S_{E}\right)$ is homotopic to $S_{E} \times 0$ in $U$ and leaves every compact subset of $M$, then $E$ is said to be simply degenerate. The sequence $\left(S_{E} \stackrel{f_{l}}{\longrightarrow} U\right)$ is called an exiting sequence. A end which is not geometrically infinite will be called geometrically finite.

THEOREM 2.4 (Hou). Let $M=\tilde{M} / \Gamma$ be a topologically tame negatively pinched 3-manifold with $\Gamma$ purely loxodromic. Then all geometrically infinite ends of $M$ are simply degenerate. And if $\Lambda(\Gamma)=S_{\infty}$, then there are no nonconstant positive superharmonic functions, or nonconstant subharmonic functions bounded above, on $M$.

3. $\Gamma$-action. In this section we will study the action of $\Gamma$ on $S_{\infty}$ and prove ergodicity of $\Gamma$ for topologically tame 3-manifolds with $\Lambda(\Gamma)=S_{\infty}$. We will prove that for such a manifold, the Green series is divergent, and that the Poincare series is also divergent if $D=2$. Theorem 1.5 will also be proved in this section.

In some situations we will take the dimension of $M$ to be 3 , otherwise we will assume $M$ is $n$-dimensional in general. 
Set the following notations throughout the paper. Let $\Gamma^{\prime} \subset \operatorname{PSL}(2, \mathbb{C})$ be a discrete orsion-free subgroup. Denote $S^{2}:=\partial \mathbb{H}^{3}$, and $S_{\infty}:=\partial \widetilde{M}$. There are many equivalent ways of equipping $S_{\infty}$ with a metric which is compatible with $\Gamma$-action. Fix a point $x \in \widetilde{M}$. Let $\xi, \zeta$ in $S_{\infty}$ be given. Set $c_{y}^{\zeta}(t)$ as the geodesic ray connecting $y$ and $\zeta$.

In [22], Gromov defined a metric on $S_{\infty}$ as follows. For $y, z \in \widetilde{M}$, let us consider arbitrary continuous curve $c(t)$ in $\widetilde{M}$ with initial point and end point denoted by $c\left(t_{0}\right)=y$ and $c\left(t_{1}\right)=z$ respectively. Define a nonnegative real-valued function $\mathcal{G}_{x}$ on $\widetilde{M} \times \widetilde{M}$ by

$$
\mathcal{G}_{x}(y, z):=\inf _{\text {all } c}\left(\int_{\left[t_{0}, t_{1}\right]} e^{-\operatorname{dist}(x, c(t))} \mathrm{d} t\right) .
$$

In particular, Gromov showed the function $\mathcal{G}_{x}$ extends continuously to $S_{\infty} \times S_{\infty}$. Every element of $\Gamma$ extends to $S_{\infty}$ as a Lipschitz map with respect to $\mathcal{G}_{x}$.

In [31] the following metrics are shown to be equivalent to the Gromov's metric.

$K_{x}$ metric : Let $B_{\zeta}$ denote the Busemann function based at $x_{0}$. Set $B_{\zeta}(x, y)$ $=B_{\zeta}(x)-B_{\zeta}(y)$, for $x, y \in \widetilde{M}$, the function $B_{\zeta}(x, y)$ is called the Busemann cocycle. Define $\beta_{x}: S_{\infty} \times$ $S_{\infty} \longrightarrow \mathbb{R}$ by $\beta_{x}(\xi, \zeta):=B_{\xi}(x, y)+B_{\zeta}(x, y)$ where $y$ is a point on the geodesic connecting $\xi$ and $\zeta$. The $K_{x}$ metric is then defined by

$$
K_{x}(\xi, \zeta):=e^{-\frac{1}{2} \beta_{x}(\xi, \zeta)}
$$

$L_{x}$ metric: Let $\alpha_{x}(\xi, \zeta)$ denote the distance between $x$ and the geodesic connecting $\xi$ and $\zeta$. The function $L_{x}: S_{\infty} \times S_{\infty} \longrightarrow \mathbb{R}$ is then defined by

$$
L_{x}(\xi, \zeta):=e^{-\alpha_{x}(\xi, \zeta)}
$$

$d_{x}$ metric: Define a function $l_{x}: S_{\infty} \times S_{\infty} \longrightarrow \mathbb{R}$ by $l_{x}(\xi, \zeta):=\sup \{\tau \mid$ $\left.\operatorname{dist}\left(c_{\xi}^{x}(\tau), c_{\zeta}^{x}(\tau)\right)=1\right\}$. Geometrically, a neighborhood about $\xi$ in $S_{\infty}$ with respect to the topology induced by $l_{x}$ is the shadow cast by the intersection of 1-ball about $c_{x}^{\xi}(\tau)$ and $\tau$-sphere about $x$. The $d_{x}$ metric is then defined by

$$
d_{x}(\xi, \zeta):=e^{-l_{x}(\xi, \zeta)}
$$

It was originally observed for symmetric spaces by Mostow [36] that the boundary map is quasi-conformal. This property continue to hold in negatively curved spaces, see [25] and [40]. Here we give a proof of this fact with respect to the above metrics.

Proposition 3.1. Let $h$ be a quasiisometry between two negatively pinched curved spaces. The boundary extension map $\bar{h}$ is quasi-conformal on the boundary with respect to $d_{x}, L_{x}, K_{x}, \eta_{v}$-metrics.

Proof. For the proof of $\eta_{v}$-metric See Proposition 3.1 in [25]. Fix $x \in \tilde{M}$. Let us take $d_{x}$-metric. Set $\lambda \geq L$. Denote by $S(x ; y, R)$ the shadow cased from $x$ of the metric sphere $S(y, R)$ with center located at $y$ and radius $R$, i.e. $S(x ; y, R)=$ $\left\{\xi \in S_{\infty} \mid c_{x}^{\xi} \cap S(y, R) \neq \emptyset\right\}$. Let $B(\xi, r)$ be a ball of radius $r$ in $S_{\infty}$. Using triangle 
comparison we can show there exists a constant $\alpha_{b} \geq 1$ depends on pinching constant $b$ such that

$$
S\left(x ; c_{x}^{\xi}\left(t_{r}\right), \lambda\right) \subset B(\xi, r) \subset S\left(x ; c_{x}^{\xi}\left(t_{r}\right), \alpha_{b} \lambda\right)
$$

for some $t_{r}>0$ which depends only on $r$. The images $\bar{\phi}\left(S\left(x ; c_{x}^{\xi}\left(t_{r}\right), \lambda\right)\right)$ and $\bar{\phi}\left(S\left(x ; c_{x}^{\xi}\left(t_{r}\right), \alpha_{b} \lambda\right)\right)$ are quasi-spheres, i.e. there exists a constant $\beta_{\phi}>0$ depends on $\phi$ such that $S\left(\bar{\phi}(x) ; \bar{\phi}\left(c_{x}^{\xi}\left(t_{r}\right)\right), \beta_{\phi}^{-1} \lambda\right) \subset \bar{\phi}\left(S\left(x ; c_{x}^{\xi}\left(t_{r}\right), \lambda\right)\right)$ and $\bar{\phi}\left(S\left(x ; c_{x}^{\xi}\left(t_{r}\right), \alpha_{b} \lambda\right) \subset\right.$ $S\left(\bar{\phi}(x) ; \bar{\phi}\left(c_{x}^{\xi}\left(t_{r}\right)\right), \beta_{\phi} \alpha_{b} \lambda\right)$. On the other hand, by estimates in [11] there exists positive numbers $A_{1}\left(\beta_{\phi}, \lambda\right)$ and $A_{2}\left(\alpha_{b}, \beta_{\phi}, \lambda\right)$ such that

$$
\begin{gathered}
B\left(\bar{\phi}(\xi), A_{1} e^{-R}\right) \subset S\left(\bar{\phi}(x) ; \bar{\phi}\left(c_{x}^{\xi}\left(t_{r}\right)\right), \beta_{\phi}^{-1} \lambda\right), \\
S\left(\bar{\phi}(x) ; \bar{\phi}\left(c_{x}^{\xi}\left(t_{r}\right)\right), \beta_{\phi} \alpha_{b} \lambda\right) \subset B\left(\bar{\phi}(\xi), A_{2} e^{-R}\right)
\end{gathered}
$$

where $R=\operatorname{dist}\left(\bar{\phi}(x), \bar{\phi}\left(c_{x}^{\xi}\left(t_{r}\right)\right)\right.$. Hence the result follows by setting $r_{\phi}(\xi, r)=A_{1} e^{-R}$ and $\kappa=A_{2} / A_{1}$.

Proposition 3.2. Let $f: \partial \tilde{N} \longrightarrow S_{\infty}$ be a embedding conjugate $\Gamma_{1}$ to $\Gamma_{2}$ under isomorphism $\chi: \Gamma_{1} \longrightarrow \Gamma_{2}(f \circ \gamma=\chi(\gamma) \circ f)$. Then $f\left(\Lambda_{\Gamma_{1}}\right)=\Lambda_{\Gamma_{2}}$.

Proof. Let $\gamma \in \Gamma_{1}$. Since $\gamma f^{-1}\left(\Lambda_{\Gamma_{2}}\right)=f^{-1}\left(\chi(\gamma) \Lambda_{\Gamma_{2}}\right)$, and by $\Gamma_{2}$-invariance of $\Lambda_{\Gamma_{2}}$, we have $f^{-1}\left(\Lambda_{\Gamma_{2}}\right)$ is $\Gamma_{1}$-invariant closed set. Note that $f^{-1}\left(\Lambda_{2}\right)$ is nonempty, since fixed points of elements of $\Gamma_{1}$ are also fixed points of elements of $\Gamma_{2}$, hence $f\left(\Lambda_{\Gamma_{1}}\right) \subseteq \Lambda_{\Gamma_{2}}$. Similarly we also have $f\left(\Lambda_{\Gamma_{1}}\right) \supseteq \Lambda_{\Gamma_{2}}$, and result follows.

Proposition 3.3. Let $\Gamma$ be a topologically tame, torsion-free, discrete subgroup of $\operatorname{ISO}(\widetilde{M})$ with $\Lambda_{\Gamma}=S_{\infty}$. Let $\Gamma^{\prime}$ be a topologically tame, discrete subgroup of $\operatorname{PSL}(2, \mathbb{C})$. Suppose $f: S_{\infty} \longrightarrow S^{2}$ is a homeomorphism conjugate $\Gamma$ to $\Gamma^{\prime}$. Then $D_{\Gamma^{\prime}}=2$ and $\Gamma^{\prime}$ is divergent.

Proof. By Proposition 3.2 the hyperbolic manifold $N=\mathbb{H}^{3} / \Gamma^{\prime}$ is topologically tame and $\Lambda_{\Gamma^{\prime}}=S^{2}$. It follows from analytical tameness and Theorem 9.1 of [10], there exists no non-trivial positive superharmonic function on $N$ with respect to the hyperbolic Laplacian $\Delta$. Let $P(y, \xi)$ denote the Poisson kernel on $\mathbb{H}^{3}$. The $D_{\Gamma^{\prime}}$ dimensional conformal measure (Patterson-Sullivan measure, see end of §3) $\sigma_{y}$ has Radon-Nikodym derivative of $P(y, \xi)^{D_{\Gamma^{\prime}}}$, i.e. $\frac{\mathrm{d} \sigma_{y}}{\mathrm{~d} \gamma^{*} \sigma_{y}}(\xi)=P\left(\gamma^{-1} y, \xi\right)^{D_{\Gamma^{\prime}}}$. The $\Gamma^{\prime}$ invariant function $h(y):=\sigma_{y}\left(S^{2}\right)$ satisfies $\Delta h=D_{\Gamma^{\prime}}\left(D_{\Gamma^{\prime}}-2\right) h$, which implies $h$ is non-trivial superharmonic if $D_{\Gamma^{\prime}} \neq 2$. And it follows that $\Gamma^{\prime}$ must also be divergent.

Let $C$ be a subset of $S_{\infty}$. Let $\lambda$-dimensional Hausdorff measure of $C$ on the metric space $\left(S_{\infty}, \rho_{x}\right)$ be denoted by $\mathfrak{M}_{\rho_{x}}^{\lambda}(C)$. Observe that for any $x \in \widetilde{M}$ and any $\gamma \in \Gamma$, we have $\gamma^{*} \mathfrak{M}_{K_{x}}^{\lambda}=\mathfrak{M}_{K_{\gamma^{-1}}}^{\lambda}$; this follows from the straightforward identity.

A family of finite Borel measures $\left[\nu_{y}\right]_{y \in \widetilde{M}}$. will be called a $\lambda$-conformal density under the action of $\Gamma$ if for every $x \in \widetilde{M}$ and every $\gamma \in \Gamma$ we have $\gamma^{*} \nu_{y}=\nu_{\gamma^{*} y}$, and the Radon-Nikodym derivative $\frac{\mathrm{d} \nu_{y}}{\mathrm{~d} \gamma^{*} \nu_{y}}(\zeta)$ at any point $\zeta \in S_{\infty}$ is equal to $e^{-\lambda B_{\zeta}\left(\gamma^{-1} y, y\right)}$. (This is to be interpreted as being vacuously true if, for example, the measures in 
the family are all identically zero). Although there can not be any $\Gamma$-invariant nontrivial finite Borel measure on $\Lambda_{\Gamma}$ for non-elementary $\Gamma$, we can always define a $\Gamma$ invariant non-trivial locally finite measure $\Pi_{\nu_{x}}$ on $\Lambda_{\Gamma} \times \Lambda_{\Gamma}$ by setting $\mathrm{d} \Pi_{\nu_{x}}(\xi, \zeta)=$ $e^{\lambda \beta_{x}(\xi, \zeta)} \mathrm{d} \nu_{x}(\xi) \mathrm{d} \nu_{x}(\zeta)$. The measure $\Pi_{\nu_{x}}$ corresponds to the Bowen-Margulis measure on the unit tangent bundle $S \widetilde{M}$ see [31].

Let us recall a fundamental fact about conformal density, which was originally proved by Sullivan for $\Gamma \subset \mathrm{SO}(n, 1)$ and generalized to the pinched negatively curved spaces in [46]. It relates the divergence of $\Gamma$ at the critical exponent $D_{\Gamma}$ with ergodicity of the $D_{\Gamma}$-conformal density under the action of $\Gamma$.

We will say that two Borel measures on $S_{\infty}$ are in the same $\Gamma$-class if the RadonNikodym derivative of $\gamma^{*} \nu_{1}$ with respect to $\nu_{1}$ is equal to the Radon-Nikodym derivative of $\gamma^{*} \nu_{2}$ with respect to $\nu_{2}$.

Proposition 3.4 (see [46]). Let $\Gamma$ be a nonelementary, discrete, torsion-free and divergent at $D_{\Gamma}$. Suppose $[\nu]$ is a $D_{\Gamma}$-conformal density under the action of $\Gamma$, then $\Gamma$ act ergodically on $\Lambda_{\Gamma}$ and $\Lambda_{\Gamma} \times \Lambda_{\Gamma}$ with respect to $\left[\nu_{x}\right]$ and $\left[\Pi_{\nu_{x}}\right]$ respectively.

Proposition 3.5 (see [37]). Let $\Gamma$ be nonelementary and discrete. Suppose that $\Gamma$ acts ergodically on $S_{\infty}$ with respect to a measure $\nu$ defined on $S_{\infty}$. Then every measure of $S_{\infty}$ in the same measure class as $\nu$ is a constant multiple of $\nu$.

Proposition 3.6. Let $\Gamma$ be a non-elementary discrete subgroup of the isometry group of $\tilde{M}$. If $\left[\nu_{y}\right]_{y \in \tilde{M}}^{D}$ is a non-trivial $\Gamma$-invariant $D$-conformal density, then $D \neq 0$.

Proof. Suppose $D=0$. Then $\nu_{y}$ is a $\Gamma$-invariant non-trivial finite Borel measure. Since $\Gamma$ is non-elementary, there exists a loxodromic element $\gamma$ in $\Gamma$. Let $\xi, \zeta \in S_{\infty}$ be the two distinct fixed points of $\gamma$. Let $\langle\gamma\rangle$ be the group generated by $\gamma$. Then $\nu_{y}$ is clearly $\langle\gamma\rangle$-invariant. But $\gamma$ is loxodromic, so we must have $\operatorname{supp}\left(\nu_{y}\right) \subset\{\xi, \zeta\}$. Then, by the fact that $\Lambda(\Gamma)$ is infinite, we have $\nu_{y}$ is an infinite measure, which is a contradiction.

Proposition 3.7. Let $\Gamma$ be a discrete subgroup of $\operatorname{ISO}(\widetilde{M})$. Suppose $\mathfrak{M}_{K_{x}}^{\lambda}$ is a finite measure. Then $\mathfrak{M}_{K_{x}}^{\lambda}$ is a $\lambda$-conformal density under the action of $\Gamma$.

There is a canonical way of constructing $D_{\Gamma}$-dimensional conformal density which is due to Patterson-Sullivan as follows; By applying a adjusting function we can always assume the Poincaré series diverges at $D_{\Gamma}$. The measures

$$
\mu_{x, s}:=\frac{\sum_{\gamma \in \Gamma} e^{-s \operatorname{dist}(z, \gamma x)} \delta_{\gamma x}}{\sum_{\gamma \in \Gamma} e^{-s \operatorname{dist}(z, \gamma z)}} \quad ; s>D_{\Gamma}
$$

converges weakly to a limiting measure $\mu_{x}$ as $s_{n} \rightarrow D_{\Gamma}$ through a subsequence. It is trivial to see that $\mu_{x}$ is supported on $\Lambda_{\Gamma}$. The measure $\left[\mu_{x}\right]$ is called Patterson-

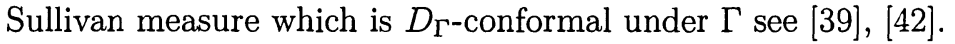

From now on for $\Gamma^{\prime} \subset \operatorname{PSL}(2, \mathbb{C})$, we will denote the Patterson-Sullivan measure on $\Lambda_{\Gamma^{\prime}}$ by $\sigma_{y}$.

Let $\Lambda_{\Gamma}^{c}$ denote the set of conical limit points in $\Lambda_{\Gamma}$. Recall a point $\xi \in \Lambda_{\Gamma}$ is in $\Lambda_{\Gamma}^{c}$ if and only if there exists $\left\{\gamma_{n}\right\} \subset \Gamma$ such that $\operatorname{dist}\left(\gamma_{n} c_{x}^{\xi}\left(t_{n}\right), x\right)<c$ for some $c>0$ and sequence of $t_{n}$. Obviously $\Lambda_{\Gamma}^{c}$ is $\Gamma$-invariant, and non-empty (a loxodromic fixed 
points are in $\Lambda_{\Gamma}^{c}$ ), hence it is a dense $\Gamma$-invariant subset of $\Lambda_{\Gamma}$. A equivalent definition for the conical limit point $\xi$ is that it must be contained in infinitely many shadows $S\left(x ; \gamma_{n} x, c\right)$. Hence $\Lambda_{\Gamma}^{c}=\cup_{\lambda>0} \cap_{m \geq 1} \cup_{n>m} S\left(x, \gamma_{n} x, \lambda\right)$. It is a easy fact from the construction of $\mu_{x}$, no points in $\Lambda_{\Gamma}^{c}$ can be a atom for $\mu_{x}$, and if $\operatorname{supp}\left(\mu_{x}\right) \subseteq \Lambda_{\Gamma}^{c}$ then $\Gamma$ is divergent. In fact it is a deep result of Sullivan that $\Gamma$ is divergent if and only if $\operatorname{supp}\left(\mu_{x}\right) \subseteq \Lambda_{\Gamma}^{c}$.

LemMa 3.8 (see [12]; Sullivan's Shadow Lemma). Let $\mu_{x}$ be a $D_{\Gamma}$-conformal density with respect to $\Gamma$, which is not a single atom. Then there exists constants $\alpha>0$ and $\lambda_{o} \geq 0$, such that,

$$
\alpha^{-1} e^{-D_{\Gamma} \operatorname{dist}\left(x, \gamma^{-1} x\right)} \leq \mu_{x}(S(x ; \gamma x, \lambda)) \leq \alpha e^{-D_{\Gamma} \operatorname{dist}\left(x, \gamma^{-1} x\right)+2 D \lambda},
$$

for all $\gamma \in \Gamma$ and $\lambda \geq \lambda_{o}$.

Proposition 3.9. Let $\Gamma \subset \operatorname{ISO}(\widetilde{M})$ be a discrete subgroup. Suppose either $\Lambda_{\Gamma}=\Lambda_{\Gamma}^{c}$ or $\Lambda_{\Gamma}=S_{\infty}$ and $\Gamma$ is divergent. Then $\mu_{x}$ is positive on all non-empty relative open subsets of $\Lambda_{\Gamma}$.

Proof. Suppose $\Lambda_{\Gamma}=S_{\infty}$. It suffices to show $\mu_{x}$ is positive for any non-empty open ball $B(\xi, r)$ with respect to the $d_{x}$-metric. Fix $\lambda>\lambda_{o}$. Let $\zeta \in \Lambda_{\Gamma}^{c} \cap B(\xi, r)$ (note that $\Lambda_{\Gamma}^{c}$ is dense in $\Lambda_{\Gamma}$ so the intersection is nonempty). Then we can choose $\gamma \in \Gamma$ such that $S(x ; \gamma x, \lambda) \subset B(\xi, r)$. By assumption $\Gamma$ is divergent, we have $\operatorname{supp}\left(\mu_{x}\right) \subseteq$ $\Lambda_{\Gamma}^{c}$. Since no points of $\Lambda_{\Gamma}^{c}$ can be a atom for conformal density, the result follows from Lemma 3.8. Same argument works if $\Lambda_{\Gamma}=\Lambda_{\Gamma}^{c} \mathrm{u}$

Let us define a function $\Theta: \tilde{M} \times \tilde{M} \times S_{\infty} \longrightarrow \mathbb{R}^{+}$by $\Theta(x, y, \xi):=\exp \left(-B_{\xi}(x, y)\right)$.

\section{Harmonic Density}

Let $\lambda_{1}$ and $\tilde{\lambda}_{1}$ denote the first of the spectrum of $\Delta$ on $M=\tilde{M} / \Gamma$, and of $\tilde{\Delta}$ on $\tilde{M}$, respectively. Recall that for a noncompact open manifold, the first of the spectrum is defined as

$$
\lambda_{1}:=\inf _{f \in C_{o}^{\infty}, f \neq 0}\left(\frac{\int|\nabla f|^{2}}{\int f^{2}}\right)
$$

where $C_{o}^{\infty}$ is the space of smooth functions on $M$ with compact support. Note that we always have $\lambda_{1} \leq \tilde{\lambda}_{1}$.

The $\lambda_{1}$-harmonic functions has been studied by Ancona in [2] and [3].

Proposition 3.10 (Ancona). For each $s<\lambda_{1}$, the elliptic operator $\tilde{\Delta}+s I$ has a Green function $G_{s}(x, y)$, and there exists a function $f: \mathbb{R}^{+} \longrightarrow \mathbb{R}^{+}$such that $\sum_{\gamma \in \Gamma} \hat{G}_{s}(x, \gamma y)$ converges for $s<\lambda_{1}$ and diverges for $s \geq \lambda_{1}$, where $\hat{G}_{s}(x, \gamma y):=$ $\exp (f(\operatorname{dist}(y, \gamma y))) G_{s}(x, \gamma y)$. Furthermore, $\mathfrak{P}_{s}(x, y, \zeta):=\lim _{z \rightarrow \zeta} \frac{G_{s}(x, z)}{G_{s}(y, z)}$ defines the Poisson kernel of $\tilde{\Delta}+$ sI at $\zeta \in S_{\infty}$.

Similarly to the construction of $\mu_{x}$ from $Z_{\Gamma}$, one can also construct a family of Borel measures from $\sum_{\gamma \in \Gamma} \hat{G}_{s}(x, \gamma y)$.

Proposition 3.11. Let $x$ be any point of $\tilde{M}$. There exists a family of Borel measures $\left[\omega_{y}^{1}\right]_{y \in \tilde{M}}$ on $S_{\infty}$ such that (i) for all $x, y \in \tilde{M}$, Radon-Nikodym derivative $\mathrm{d} \omega_{y}^{1} / \mathrm{d} \omega_{x}^{1}$ at any point $\zeta \in S_{\infty}$ is equal to $\mathfrak{P}_{\lambda_{1}}(x, y, \zeta)$ and $(i i) \omega_{x}^{1}$ is of mass 1 . 
Let us denote the harmonic density of $\tilde{\Delta}$ by $\left[\omega_{y}\right]_{y \in \tilde{M}}$ with $\omega_{x}$ normalized of mass 1. By definition this means that every harmonic function $f$ on $M$ with boundary values $f_{\infty}$ is given by

$$
f(x)=\int_{S_{\infty}} f_{\infty}(\xi) \mathrm{d} \omega_{x}(\xi)
$$

The existence and uniqueness of harmonic density follows from the solvability of the Dirichlet problem on $\tilde{M} \cup S_{\infty}$ see [1] and the Riesz Representation Theorem. The Radon-Nikodym derivative of $\omega_{x}$ at $\xi \in S_{\infty}$ is given by the Poisson kernel $\mathfrak{P}(x, y, \xi)$ of $\tilde{\Delta}$, i.e. $\frac{\mathrm{d} \omega_{y}}{\mathrm{~d} \omega_{x}}(\xi)=\mathfrak{P}(y, x, \xi)$. For any $\Gamma$-invariant subset $C \subset S_{\infty}$, the function $h_{C}$ on $\tilde{M}$ defined by $h_{C}(y):=\int_{S_{\infty}} \chi_{C} \mathfrak{P}(y, x, \xi) \mathrm{d} \omega_{x}(\xi)$ is $\Gamma$-invariant, hence defines a harmonic function on $M$.

Proposition 3.12. Let $M=\tilde{M} / \Gamma$ be a negatively pinched topologically tame 3-manifold with $\Lambda(\Gamma)=S_{\infty}$. Then $\Gamma$ is ergodic with respect to harmonic density $\left[\omega_{y}\right]_{y \in \tilde{M}}$.

Proof. Suppose not, and let $C \subset S_{\infty}$ be a $\Gamma$-invariant subset with $\omega_{x}(C)>0$ and $\omega_{x}\left(C^{c}\right)>0$. By Fatou's conical convergence theorem, we have $\chi_{C}(\xi)=$ $\lim _{t \rightarrow \infty} h_{C}\left(c_{y}^{\xi}(t)\right)$ for $\xi \in S_{\infty}$. Hence, $h_{C}$ defines a positive nonconstant $\Gamma$-invariant harmonic function, which contradicts Theorem 2.4. Therefore $\Gamma$ must be ergodic.

Proposition 3.13. Let $M$ be noncompact and satisfy the hypothesis of Proposition 3.12. Then $\omega_{x}^{1}=\omega_{x}$.

Proof. Let us note that $\lambda_{1}=0$. This follows from the fact that for a noncompact, complete Riemannian manifold $M$, if $\lambda_{1}(M)>0$ then there exists a positive Green's function $G$ on $M$. If such a $G$ exists, then $1-\exp (-G)$ defines a positive superharmonic function, which is a contradiction to Theorem 2.4. Hence we must have $\lambda_{1}=0$. Therefore $\mathfrak{P}_{\lambda_{1}}=\mathfrak{P}$, i.e $\frac{\mathrm{d} \omega_{y}^{1}}{\mathrm{~d} \omega_{x}^{1}}=\frac{\mathrm{d} \omega_{y}}{\mathrm{~d} \omega_{x}}$. Hence, by Proposition 3.12 and uniqueness, we have the desired result.

\section{Superharmonic Functions}

Let $\xi \in S_{\infty}$ be given. Let $\mathcal{E}$ be a continuous unit vector field on $\tilde{M}$ with $\mathcal{E}(x)=\Phi_{x}(\xi)$. Then by using the first length variation formula, one can show that $B_{\xi}$ is $C^{1}$ and that $-\operatorname{grad} B_{\xi}=\mathcal{E}$. In fact, the Busemann function is $C^{2, \alpha}$, see [27]. $\tilde{M}$ by

Let $\left[\mu_{y}\right]^{D}$ denote $D$-conformal density. Let us define a nonnegative function $u$ on

$$
u(y):=\int_{S_{\infty}} \Theta^{D}(y, x, \xi) \mathrm{d} \mu_{x}(\xi)
$$

Proposition 3.14. The function $u$ is a $\Gamma$-invariant and positive. It is superharmonic if $D \leq(n-1) a$, and subharmonic if $(n-1) a \leq D \leq(n-1) b$.

Proof. We can write $u(y)$ as $\mu_{y}\left(S_{\infty}\right)$. Since $u(\gamma y)=\gamma^{*} \mu_{y}\left(S_{\infty}\right)=\mu_{y}\left(S_{\infty}\right)=u(y)$ for $\gamma \in \Gamma$, we have that $u$ is $\Gamma$-invariant. 
Let $x \in \tilde{M}$ be fixed. It follows from, $\left|\nabla B_{\xi}(y, x)\right|=|\mathcal{E}|=1$ and Rauch'stheorem that we have $\exp \left(-D B_{\xi}(y, x)\right) D(D-(n-1) b) \leq \Delta \Theta^{D} \leq \exp \left(-D B_{\xi}(y, x)\right) D(D-$ $(n-1) a)$. This implies the result.

Proposition 3.15. Suppose $\Gamma$ is nonelementary, i.e. has no abelian subgroup of finite index. Suppose that there are no nontrivial $\Gamma$-invariant positive-valued superharmonic function on $\tilde{M}$. Then $(n-1) a \leq D \leq(n-1) b$.

Proof. Let $\Gamma x$ be the orbit of $x$ under $\Gamma$. Then the growth rate of the number of points of $\Gamma x$ in ball $(x, r)$ as $r$ increases is bounded by $\operatorname{vol}(\operatorname{ball}(x, r))$. By the volume comparison theorem we have $C_{n} \exp ((n-1) b r) \geq \operatorname{vol}($ ball $(x, r))$, for some constant $C_{n}$ which depends only on dimension $n$. Therefore, when $s>(n-1) b$ we get $Z_{\Gamma}(x, s)<\infty$, which implies that $D \leq(n-1) b$.

Next suppose that we have $D \leq(n-1) a$. Then by Proposition $3.14, u(x)$ is a $\Gamma$-invariant positive superharmonic and $\Delta u \leq D(D-(n-1) a) u$. It now follows from the hypothesis that $u$ is constant and that either $D=0$ or $D=(n-1) a$. However, since $\Gamma$ is nonelementary and $\left[\mu_{y}\right]$ is $\Gamma$-invariant, Proposition 3.6 implies that $D \neq 0$. Hence, $D=(n-1) a$, and the result follows.

The next proposition was originally proved by Sullivan [41] using a Borel-Cantelli type of argument. The proof is purely measure theoretic (see [37], [46]). The proposition relates the ergodicity of $\Gamma$ with the divergence of the Poincaré series at $D$.

Proposition 3.16 (Sullivan). Suppose that $\Gamma$ is nonelementary, discrete and torsion-free, and is divergent at $D$. Then $\Gamma$ is ergodic with respect to $[\mu]^{D}$.

Proposition 3.17. Suppose $D=(n-1) a$ and there are no nontrivial positive superharmonic functions on $M$. Then $\Gamma$ is divergent.

Proof. Fix a point $y \in \tilde{M}$. Let us assume the Poincare series converges at $D$ (i.e. $\left.\sum_{\gamma \in \Gamma} \exp (-(n-1) a \operatorname{dist}(x, \gamma y))<\infty\right)$. Then this series defines a nontrivial $\Gamma$-invariant function on $\tilde{M}$. Let us denote this function by $h(x)$. Since $\exp (-D \operatorname{dist}(z, \gamma y)) \leq \exp (D \operatorname{dist}(x, z)) \exp (-D \operatorname{dist}(x, \gamma y))$ for $z \in \tilde{M}$, it follows that for any given number $N>0$ there is a constant $C>0$ such that $\sum_{\gamma \in \Gamma} \exp (-D \operatorname{dist}(z, \gamma y)) \leq C \sum_{\gamma \in \Gamma} \exp (D \operatorname{dist}(x, \gamma y))$ for $\operatorname{dist}(z, x) \leq N$. Hence the series converges uniformly on compact subsets of $\tilde{M}$. We will show that the convergence of the Poincaré series at $(n-1) a$ implies existence of nontrivial positive superharmonic function on $M$.

Set $\operatorname{dist}_{\gamma y}(x):=\operatorname{dist}(x, \gamma y)$. First, we have

$$
\Delta h(x)=\sum_{\gamma \in \Gamma} \exp \left(-D \operatorname{dist}_{\gamma y}(x)\right) D\left(D\left|\nabla \operatorname{dist}_{\gamma y}(x)\right|^{2}-\Delta \operatorname{dist}_{\gamma y}(x)\right) .
$$

By Rauch'stheorem and $\mid \nabla$ dist $\left._{\gamma y}(x)\right|^{2}=1$ we get

$$
\Delta h(x) \leq \sum_{\gamma \in \Gamma} \exp (-D \operatorname{dist}(x, \gamma y)) D(D-(n-1) a)
$$

which implies $\Delta h \leq 0$. We consider the series $\sum_{\gamma \in \Gamma} \log \tanh \left(\frac{(n-1) a \text { dist }_{\gamma y}(x)}{2}\right)$. It is easy to see that the convergence of this series on the set of points bounded away from 
$\Gamma y$ follows from the convergence of the Poincare series at $D=(n-1) a$. Denote this series by $-f_{\tilde{\alpha}}$. Then by direct computation and Rauch's theorem we have $\Delta f(x) \leq$ 0 for $x \in \tilde{M} \backslash \Gamma y$. Hence $1-\exp (-f(x))$ defines a nontrivial positive $\Gamma$-invariant superharmonic function on $\tilde{M}$.

Therefore, the convergence of the Poincaré series at $D=(n-1) a$ give raise to contradictions to our hypothesis, and the result follows.

Corollary 3.18. Suppose $D=(n-1) a$ and there are no nontrivial positive superharmonic functions on $M$. Then, $\Gamma$ is ergodic with respect to $[\mu]^{D}$.

Proof. The corollary follows from Proposition 3.16 and Proposition 3.17.

Corollary 3.19. Let $M=\tilde{M} / \Gamma$ be a topologically tame 3 -manifold with $-b^{2} \leq$ $\mathcal{K} \leq-1$ and $\Lambda(\Gamma)=S_{\infty}$. If $D=2$, then $\Gamma$ is divergent, hence ergodic with respect to $[\mu]^{D}$.

Proof. The corollary follows from Theorem 2.4, Proposition 3.17 and Corollary 3.18.

Proof. [Proof of Theorem 1.5] Under the hypothesis of Theorem 1.5, it follows from Proposition 3.15 that $D \in[2,2 b]$. That $\Gamma$ is harmonically ergodic follows from Proposition 3.12. If $D=2$, then by Corollary 3.19 we have $\Gamma$ is divergent.

4. Part I of Theorems 1.1 and 1.2. Let $\Gamma$ be a torsion-free discrete subgroup of $\operatorname{ISO}(\widetilde{M})$ with $D_{\Gamma}=2$. We assume $\Gamma$ is either convex-cocompact or $\Lambda_{\Gamma}=S_{\infty}$, hausdorff-conservative and divergent.

Proposition 4.1. The measure $\mathfrak{M}_{K_{x}}^{2}$ is finite and positive on all non-empty relative open subsets of $\Lambda_{\Gamma}$, and $\mathfrak{M}_{K_{x}}^{2}(A)=0$ if and only if $\mathfrak{M}_{\eta_{v}}^{2}(A)=0$ for $A \subset$ $\Lambda_{\Gamma} \backslash v(-\infty)$

Proof. First note that if we replace dist ${ }_{v, t}$ with dist in the definition of $\eta_{v}$ we get a equivalent metric by Lemma 4 in [26].

Let $x \in \widetilde{M}$ be any point. Denote $H_{v, x}$ the horosphere tangent to $v(\infty)$ and passing through $x$. Take two vectors $U^{\zeta}, U^{\xi}$ in $S \widetilde{M}$ that are asymptotic to $v(-\infty)$ and passing through $H_{v, x}$ with $U^{\zeta}(\infty)=\zeta$ and $U^{\xi}(\infty)=\xi$. Then there exists a positive constant $\alpha$ such that for any unite tangent vectors $v^{\zeta}, v^{\xi}$ at $x$ which are asymptotic to $\zeta$ and $\xi$ respectively, we have $\operatorname{dist}\left(g_{t} U^{\zeta}, g_{t} v^{\zeta}\right) \leq \alpha e^{-t}$ and $\operatorname{dist}\left(g_{t} U^{\xi}, g_{t} v^{\xi}\right) \leq \alpha e^{-t}$, where $g_{t}$ is the flow. This gives $\operatorname{dist}\left(g_{\tau} U^{\zeta}, g_{\tau} U^{\xi}\right) \leq 2 \alpha e^{-\tau}+1$ with $\tau=l_{x}(\zeta, \xi)$. On the other hand we also have $\beta^{-1} e^{t} \leq \operatorname{dist}\left(g_{t} U^{\zeta}, g_{t} U^{\xi}\right) \leq \beta e^{b t}$ for some positive constant $\beta$, which gives $e^{-s} \geq \beta^{-1}$ and $e^{-s} \leq \beta^{1 / b}$ when $\operatorname{dist}\left(g_{s} U^{\zeta}, g_{s} U^{\xi}\right)=1$. Hence $\frac{\beta^{-1} \beta^{1 / b}}{2 \alpha+1} e^{-s} \leq e^{-\tau} \leq \beta e^{-s}$. Therefore $\eta_{v}$ and $K_{x}$ are equivalent on all points in $S_{v, x}$, where $S_{v, x}$ is the shadow of $H_{v, x}$ cased from $v(-\infty)$. By compactness of $S_{\infty}$ there are $\left\{v_{1}, \ldots, v_{n}\right\} \subset S \widetilde{M}$ such that $\cup_{1}^{n} S_{v_{i}, x}=S_{\infty}$. Since $0<\mathfrak{M}_{\eta_{v_{i}}}^{2}\left(S_{v_{i}, x} \cap \Lambda_{\Gamma}\right)<\infty$, we have $\mathfrak{M}_{K_{x}}^{2}$ is positive and finite on $\Lambda_{\Gamma}$. It follows from Propositions 3.7, 3.5 and 3.9 the measure $\mathfrak{M}_{K_{x}}^{2}$ is positive on all relative open subsets. Let $A \subset S_{\infty} \backslash v(-\infty)$ be a $\mathfrak{M}_{\eta_{v}}^{2}$-null set. Let $\delta>0$. Note that $\cup_{x \in \widetilde{M}} S_{v, x}=S_{\infty} \backslash v(-\infty)$. Hence there is $B \subset A$ with $B \subset S_{v, z}$ such that $\mathfrak{M}_{K_{z}}^{2}(A \backslash B)<\delta$. But $\mathfrak{M}_{K_{z}}^{2}(B) \leq c \mathfrak{M}_{\eta_{v}}^{2}(B)$ for some $c>0$. By finiteness we have $\mathfrak{M}_{K_{z}}^{2}(A)<\delta$. Same argument holds for the rest of the proposition. 
COROLlaRY 4.2. The measures $\mu_{x}$ and $\mathfrak{M}_{\eta_{v}}^{2}$ are absolutely continuous with respect to each other. In-particular $\mathfrak{M}_{\eta_{v}}^{2}$ is supported on $\Lambda_{\Gamma}$.

Proof. The result follows from Proposition 3.7, 3.5 and Proposition 4.1.

We use Mostow and Gehring's original idea to show the regularity of quasiconformal map [36], [20]. This method was extended in [25]. We will follow their presentations, but with necessary generalizations that will allow us to prove our theorems using results from previous sections.

Take the unite ball model of $\mathbb{H}^{3}$. Let $u$ be a unit tangent vector at the origin. Let $\mathcal{O}_{u}$ be the unit circle on $\partial \mathbb{H}^{3}=S^{2}$ which is contained in the unique totally geodesic plane perpendicular to $u$ and passing through the origin. Also denote the point $u(\infty)$ on $S^{2}$ by $\varsigma$. Then for any pair $(p, \varsigma) \in \mathbb{B}_{u}:=\mathcal{O}_{u} \times \varsigma$ there is a unique semi-circle connecting them. The bundle of all these semi-circles is the upper hemisphere $\Omega_{u}$ of $S^{2}$. We denote this bundle space by $\left(\Omega_{u}, \pi_{u}, \mathbb{B}_{u}\right)$

where $\pi_{u}$ is the projection.

Let $\phi: S^{2} \longrightarrow S_{\infty}$ be a quasi-conformal embedding conjugate $\Gamma^{\prime}$ to $\Gamma$ under isomorphism $\chi: \Gamma^{\prime} \longrightarrow \Gamma$, here $\Gamma^{\prime}$ is a topologically tame, torsion-free, discrete subgroup of $\operatorname{PSL}(2, \mathbb{C})$ with $\Lambda_{\Gamma^{\prime}}=S^{2}$. And let $\psi$ be the inverse of $\phi$ when it is a quasi-conformal homeomorphism.

Let $\rho_{u}$ be the metric on $S^{2} \backslash u(-\infty)$ which is defined same as $\eta_{v}$ with $v(-\infty)=$ $\phi(u(-\infty))$. The hausdorff measure $\mathfrak{M}_{\rho_{u}}^{2}$ on $S^{2} \backslash u(-\infty)$ with respect to $\rho_{u}$-metric is the usual Lebesgue measure. Hence there exists a constant $\omega>0$ such that for all $\theta \in S^{2} \backslash u(-\infty)$, we have $\mathfrak{M}_{\rho_{u}}^{2}\left(B_{\rho_{u}}(\theta, r)\right)=\omega r^{2}$.

Proposition 4.3 (see [36], [25]). The measure $\phi^{*} \mathfrak{M}_{\eta_{v}}^{1}$ is absolutely continuous with respect to measure $\mathfrak{M}_{\rho_{u}}^{1}$ on semi-circles. Here $\mathfrak{M}_{\eta_{v}}^{1}$ and $\mathfrak{M}_{\rho_{u}}^{1}$ are 1-dimensional hausdorff measures with respect to the $\eta_{v}$-metric and $\rho_{u}$-metric respectively.

Proof. Let $\mathfrak{L}$ be the Lebesgue measure on $\mathbb{B}_{u}$. Then for all $P \in \mathbb{B}_{u}$ we have the following derivative

$$
\lambda(P):=\lim _{r \rightarrow 0} \frac{\mathfrak{M}_{\eta_{v}}^{2}\left(\bar{\phi} \circ \pi_{u}^{-1}\left(B_{\rho_{u}}(P, r) \cap \mathbb{B}_{u}\right)\right)}{\mathfrak{L}\left(B_{\rho_{u}}(P, r) \cap \mathbb{B}_{u}\right)}
$$

exists and finite for $\mathfrak{L}$-almost everywhere, see [18].

Choose $P \in \mathbb{B}_{u}$ with $\lambda(P)<\infty$. For a semi-circle $l:=\pi_{u}^{-1}(P)$, let $U_{r}(l)$ denote the $r$-neighborhood of $l$, then $\limsup _{r \rightarrow 0} \mathfrak{M}_{\eta_{v}}^{2}\left(\bar{\phi}\left(U_{r}(l)\right)\right) / r<\infty$. For any compact $K \subset l$ with $\mathfrak{M}_{\rho_{u}}^{1}(K)=0$, choose a number $C>0$ with $\mathfrak{M}_{\eta_{v}}^{2}\left(\bar{\phi}\left(U_{r}(l)\right)\right) / r<C$. Let $\epsilon>0$ be given, by Besicovic's covering theorem there exists $\left\{\theta_{1}, \ldots, \theta_{k}\right\} \subset K$ such that $k r<\epsilon, K \subset \cup_{1}^{k} B_{\rho}\left(\theta_{i}, r\right)$ and any three of the balls $B_{\rho}\left(\theta_{i}, r\right)$ with distinct centers are disjoint.

Let $s_{i}:=\inf \left\{s>0 \mid \bar{\phi}\left(B_{\rho}\left(\theta_{i}, r\right)\right) \subset B_{\eta}\left(\bar{\phi}\left(\theta_{i}\right), s\right)\right\}$ and $\kappa>0$ (conformal constant) provided by Proposition 3.1. Then we have $\bar{\phi}(K) \subset \cup_{1}^{k} B_{\eta}\left(\bar{\phi}\left(\theta_{i}\right), s_{i}\right)$, $\bar{\phi}\left(S^{2}\right) \cap B_{\eta}\left(\bar{\phi}\left(\theta_{i}\right), s_{i} / \kappa\right) \subset \bar{\phi}\left(B_{\rho}\left(\theta_{i}, s_{i}\right)\right)$. Since $\Gamma$ is hausdorff-conservative and by Proposition 3.2, Corollary 4.2, there exists $\alpha>0$ such that

$$
\begin{aligned}
\left(\sum_{1}^{k} s_{i}\right)^{2} & \leq k \sum_{1}^{k} s_{i}^{2} \leq k \kappa^{2} \alpha \sum_{1}^{k} \mathfrak{M}_{\eta_{v}}^{2}\left(\bar{\phi}\left(B_{\rho}\left(\theta_{i}, r\right)\right)\right) \\
& \leq 2 \kappa^{2} \alpha k \mathfrak{M}_{\eta_{v}}^{2}\left(\bar{\phi}\left(U_{r}(K)\right)\right) \leq 2 \kappa^{2} \alpha k \mathfrak{M}_{\eta_{v}}^{2}\left(\bar{\phi}\left(U_{r}(l)\right)\right) \\
& \leq 2 \kappa^{2} C \alpha(k r) \leq \mathrm{const} \quad \epsilon .
\end{aligned}
$$


Note the fact that any three of $\bar{\phi}\left(B_{\rho}\left(\theta_{i}, r\right)\right)$ do not intersect is used to bound $\sum_{1}^{k} \mathfrak{M}_{\eta_{v}}^{2}\left(\bar{\phi}\left(B_{\rho}\left(\theta_{i}, r\right)\right)\right)$ by $2 \mathfrak{M}_{\eta_{v}}^{2}\left(\bar{\phi}\left(U_{r}(K)\right)\right)$.

Therefore the result follows from the last inequality.

The balls $B_{\rho}(\theta, r), \theta \in S^{2} \backslash u(-\infty), r>0$ form a Vitali relation for the Lebesgue measure $\mathfrak{M}_{\rho_{u}}^{2}$. The following derivative

$$
J(\theta):=\lim _{r \rightarrow 0} \frac{\mathfrak{M}_{\eta_{v}}^{2}\left(\phi\left(B_{\rho}(\theta, r)\right)\right)}{\mathfrak{M}_{\rho}^{2}\left(B_{\rho_{u}}(\theta, r)\right)}
$$

exists and finite for $\mathfrak{M}_{\rho}^{2}$-almost every $\theta \in S^{2} \backslash u(-\infty)$.

Proposition 4.4. Let $\operatorname{Lip}_{\phi}$ be defined by $\operatorname{Lip}_{\phi}: \theta \longrightarrow \lim \sup _{r \rightarrow 0} r_{\phi}(\theta, r) / r$. Then $\operatorname{Lip}_{\phi} \in L_{\text {loc }}^{2}\left(S^{2} \backslash u(-\infty), \mathfrak{M}_{\rho_{u}}^{2}\right)$. In-fact there exists a constant $k>0$ such that

$$
\sqrt{J(\theta)} / k \leq \lim \inf _{r \rightarrow 0} r_{\phi}(\theta, r) / r \leq \lim \sup _{r \rightarrow 0} r_{\phi}(\theta, r) / r \leq k \sqrt{J(\theta)} .
$$

Proof. Let $\epsilon>0$. There is $r_{\epsilon}>0$ such that for any $r<r_{\epsilon}$ we have

$$
\omega f(\theta) r^{2} / 2 \leq \mathfrak{M}_{\eta_{v}}^{2}\left(\bar{\phi}\left(B_{\rho}(\theta, r)\right)\right) \leq(2 \omega f(\theta)+\epsilon) r^{2}
$$

where the fact that $\mathfrak{M}_{\rho_{u}}^{2}$ is Lebesgue measure, i.e. $\mathfrak{M}_{\rho_{u}}^{2}\left(B_{\rho_{u}}(\theta, r)\right)=\omega r^{2}$ for some constant $\omega>0$ has been used. Since $\Gamma$ is hausdorff-conservative and by Proposition 3.2 , Corollary 4.2 , there exists some constant $\alpha>0$ such that

$$
\left(r_{\phi}(\theta, r) / \beta\right)^{2} / \alpha \leq \mathfrak{M}_{\eta}^{2}\left(\bar{\phi}\left(B_{\rho}(\theta, r)\right)\right) \leq \alpha\left(r_{\phi}(\theta, r)\right)^{2} .
$$

Hence we have

$$
\sqrt{(\omega / 2 \alpha f(\theta))} r \leq r_{\phi}(\theta, r) \leq \sqrt{\alpha(2 f(\theta) \omega+\epsilon))} \beta r
$$

and the result follows by letting $\epsilon \rightarrow 0$.

LEMMA 4.5. The image under $\phi$ of almost every semi-circle has locally finite $\mathfrak{M}_{\eta_{v}}^{1}$-measure.

Proof. Let $f: \Omega_{u} \longrightarrow \mathbb{B}_{u} \times[0,1]$ be a diffeomorphism which maps $\pi_{u}^{-1}(P)$ over $P$ onto $P \times[0,1]$. For every compact subset $C \subset \Omega_{u}$ we can find a positive number $\alpha$ such that

- For all $x \in f(C)$ the Jacobian of $f^{-1}$ at $x$ are $<\alpha$,

- For all $P \in \mathbb{B}_{u}$ and $y \in \pi_{u}^{-1}(P) \cap C$ the local dilations at $y$ of $\left.f\right|_{\pi_{u}^{-1}(P)}$ are $<\alpha$.

Since $\bar{\phi}$ is a embedding, $\bar{\phi}\left(\Omega_{u}\right)$ is relative compact subset of $S_{\infty} \backslash v(-\infty)$ and we have by Proposition 4.4, $\int_{\Omega_{u}} \operatorname{Lip}_{\phi}^{2} \mathrm{~d}_{\mathfrak{M}_{\rho_{u}}}^{2} \leq k^{2} \mathfrak{M}_{\eta_{v}}^{2}\left(\bar{\phi}\left(\Omega_{u}\right)\right)<\infty$, and Hölder inequality gives $\int_{\Omega_{u}} \operatorname{Lip}_{\phi} \mathrm{d}_{\mathfrak{M}_{\rho_{u}}}^{2}<\infty$. Hence

$$
\begin{aligned}
\int_{\mathbb{B}_{u}}\left(\int_{\pi_{u}^{-1}(P) \cap C} \operatorname{Lip}_{\phi} \mathrm{d} \mathfrak{M}_{\eta_{v}}^{1}\right) \mathrm{d} \mathfrak{L} & \leq \alpha \int_{f(C)} \operatorname{Lip}_{\phi} \circ f^{-1} \mathrm{~d} \mathfrak{L} \mathrm{d} t \\
& \leq \alpha^{2} \int_{C} \operatorname{Lip}_{\phi} \mathrm{d}^{2} \mathfrak{M}_{\rho_{u}}^{2}<\infty
\end{aligned}
$$


where $\mathrm{d} t$ is Lebesgue measure on $[0,1]$. Now by Proposition $4.3, \bar{\phi}$ is absolutely continuous on $\pi_{u}^{-1}(P)$ therefore

$$
\mathfrak{M}_{\eta_{v}}^{1}\left(\bar{\phi}\left(\pi_{u}^{-1}(P) \cap C\right)\right) \leq \int_{\pi_{u}^{-1}(P) \cap C} \operatorname{Lip}_{\phi} \mathrm{d} \mathfrak{M}_{\rho_{u}}^{1}<\infty .
$$

Next we adapt the idea in [25] to prove the inequality part of Theorems 1.1.

Proof. [Proof. Part I of Theorems 1.1 and 1.2] For Theorem 1.2, the inequality follows from Theorem 1.5 and Proposition 3.3. Let $\Gamma$ and $\Gamma^{\prime}$ be as in Theorem 1.1 and satisfies those conditions. Note that by Proposition 3.3, $D_{\Gamma^{\prime}} \equiv 2$. Let $g$ be the Riemannian metric of $\widetilde{M}$. Set $h=\left(D_{\Gamma} / 2\right) g$ as the new metric of $\widetilde{M}$. The boundary space of $(\widetilde{M}, g)$ and $(\widetilde{M}, h)$ can be trivially identified, and $\eta_{\left(2 / D_{\Gamma}\right) v}=\eta_{v}^{D_{\Gamma} / 2}$. The critical exponent of $\Gamma$ with respect to $h$ is 2 , hence by Lemma 4.5 there is a non-trivial curve in $S_{\infty} \backslash v(-\infty)$ with finite $D_{\Gamma} / 2$-dimensional hausdorff measure with respect to $\eta_{v}$. However as noted before the curvature assumption $-b^{2} \leq K \leq-1$ of $g$ implies the $\eta_{v}$-metric is a distance on $S_{\infty} \backslash v(-\infty)$, but the distance-hausdorff dimension is $\geq 1$ for any non-trivial curves. Therefore we have $D_{\Gamma} / 2 \geq 1$.

LEMMA 4.6. Let $\Gamma^{\prime}$ be a divergent, torsion-free discrete subgroup of PSL $(2, \mathbb{C})$ with $\Lambda_{\Gamma^{\prime}}=S^{2}$ and $D_{\Gamma^{\prime}}=2$. Then the maps $\phi$ and $\psi$ are absolutely continuous with respect to $\sigma_{y}$ and $\mu_{x}$.

Proof. By ergodicity of $\Gamma, \Gamma^{\prime}$ and equivariance of $\bar{\phi}, \bar{\psi}$ and also Proposition 4.2 , its suffices to show there exists a $A \subset S^{2} \backslash u(-\infty)$ with $\mathfrak{M}_{\rho_{u}}^{2}(A)>0$ such that the Radon-Nikodym derivative of $\bar{\phi}$ at every $x \in A$ with respect to $\mathfrak{M}_{\rho_{u}}^{2}$ and $\mathfrak{M}_{\eta_{v}}^{2}$ is non-zero. Using the fact that $\eta_{v}$ is a distance function, it follows from Proposition 4.3 , for $\mathfrak{L}$-almost all $P \in \mathbb{B}_{u}$ the length of $\bar{\phi}\left(\pi_{u}^{-1}(P)\right)>0$ is bounded by $\int_{\pi_{u}^{-1}(P)} \operatorname{Lip}_{\phi} \mathrm{dM}_{\rho_{u}}^{1}$. Hence if we set $A:=\left\{x \in \Omega_{u} \mid \operatorname{Lip}_{\phi}(x)>0\right\}$, then for $\mathfrak{L}$-almost all $P \in \mathbb{B}_{u}, \mathfrak{M}_{\rho_{u}}^{1}\left(\pi_{u}^{-1}(P) \cap A\right)>0$ which implies $\mathfrak{M}_{\rho_{u}}^{2}(A)>0$. Therefore the result follows from Proposition 4.4.

5. Part II of Theorems 1.1 and 1.2. Let $\xi_{1}, \xi_{2}, \xi_{3}, \xi_{4} \in S_{\infty}$. The cross-ratio $\left|\xi_{1}, \xi_{2}, \xi_{3}, \xi_{4}\right|$ of these four points is defined as

$$
\left|\xi_{1}, \xi_{2}, \xi_{3}, \xi_{4}\right|:=\frac{e^{-\beta_{x}\left(\xi_{1}, \xi_{2}\right)} e^{-\beta_{x}\left(\xi_{3}, \xi_{4}\right)}}{e^{-\beta_{x}\left(\xi_{1}, \xi_{3}\right)} e^{-\beta_{x}\left(\xi_{2}, \xi_{4}\right)}}
$$

This definition is consistent with the hyperbolic space cross-ratio.

If $\Gamma_{1}, \Gamma_{2}$ are discrete subgroups of $\widetilde{M}$ such that both $\Gamma_{1}, \Gamma_{2}$ are divergent, and there exists a equivariant (under some group morphism $\chi$ ), nonsingular (with respect to $\mu_{1}, \mu_{2}$ Patterson-Sullivan measures on $\Lambda_{\Gamma_{1}}$ and $\Lambda_{\Gamma_{2}}$ respectively), measurable map $f: \Lambda_{\Gamma_{1}} \longrightarrow \Lambda_{\Gamma_{2}}$. Then

$$
\mathrm{d}(f \times f)^{*} \Pi_{2}(\xi, \zeta)=e^{-D_{\Gamma_{2}} \beta_{y}(f \xi, f \zeta)} g(\xi) g(\zeta) \mathrm{d} \mu_{1}(\xi) \mathrm{d} \mu_{2}(\zeta)
$$

where $g:=\frac{\mathrm{d} f^{*}\left(\mu_{2}\right)}{\mathrm{d}\left(\mu_{1}\right)}$, and $\Pi_{i}$ is the measure defined in $\S 3$ through $\mu_{i}$. From the properties of $f,(f \times f)^{*} \Pi_{2}$ is a constant $a>0$ multiple of $\Pi_{1}$. Hence $e^{D_{\Gamma_{2}} \beta_{y}(f \xi, f \zeta)} g(\xi) g(\zeta)=$ $a e^{D_{\Gamma_{1}} \beta_{x}(\xi, \zeta)}$. Therefore for $\mu_{1}$-almost everywhere we have

$$
\left|f\left(\xi_{1}\right), f\left(\xi_{2}\right), f\left(\xi_{3}\right), f\left(\xi_{4}\right)\right|=\left|\xi_{1}, \xi_{2}, \xi_{3}, \xi_{4}\right|^{D_{\Gamma_{1}} / D_{\Gamma_{2}}} .
$$


This was the idea of Sullivan for the following lemma:

LEMMA 5.1. Let $\Gamma_{1}, \Gamma_{2}$ be discrete subgroups of $\operatorname{ISO}(\widetilde{M})$ with $D_{\Gamma_{1}}=D_{\Gamma_{2}}$ and $\Gamma_{1}, \Gamma_{2}$ are divergent. Suppose there exists a equivariant nonsingular measurable map $f: \Lambda_{\Gamma_{1}} \longrightarrow \Lambda_{\Gamma_{2}}$ with respect to Patterson-Sullivan measures space $\left(\Lambda_{\Gamma_{1}}, \mu_{1}\right)$ and $\left(\Lambda_{\Gamma_{2}}, \mu_{2}\right)$. Then $f$ preserves cross-ratio $\mu_{1}$-almost everywhere.

For a finitely generated discrete subgroup $\Gamma$ of $\operatorname{PSL}(2, \mathbb{C})$. The conservative set of $\Gamma$ on $S^{2}$ coincides with $\Lambda_{\Gamma}$ up-to Lebesgue measure zero. The group $\Gamma$ is called conservative if and only if $\Lambda_{\Gamma}$ has full Lebesgue measure. Since for a topologically tame $\Gamma$, the hausdorff dimension of $\Lambda_{\Gamma}$ is equal to $D_{\Gamma}$, therefore we have the following:

Proposition 5.2. Let $\Gamma$ be a topologically tame, torsion-free discrete subgroup of $\operatorname{PSL}(2, \mathbb{C})$ with conservative $\Gamma$, then $\Gamma$ is hausdorff-conservative.

REMARK 5.3. It is a conjecture that all finitely generated discrete subgroup $\Gamma$ of $\operatorname{PSL}(2, \mathbb{C})$ are topologically tame.

Next we recall the statement of Sullivan's quasi-conformal stability for discrete subgroups of $\operatorname{PSL}(2, \mathbb{C})$.

THEOREM 5.4 (Sullivan [43]). Let $\Gamma$ be a discrete subgroup of PSL(2, $\mathbb{C})$. Then $\Gamma$ is quasi-conformally stable (i.e. if $f$ is a quasi-conformal automorphism of $S^{2}$ with $f \Gamma f^{-1} \subset \operatorname{PSL}(2, \mathbb{C})$, then $f$ is a Möbius transformation) if and only if $\Gamma$ is conservative.

COROLLARY 5.5. Let $N=\mathbb{H}^{3} / \Gamma$ be a complete hyperbolic 3-manifold for a conservative $\Gamma$. Then $N$ is quasi-isometrically stable, i.e. If there is a quasi-isometric homeomorphism $h: N \longrightarrow M$ to a hyperbolic manifold $M$, then $N$ is isometric to $M$.

Proof. [Proof. Theorem 1.2 part II] By Theorem 1.5, $\Gamma$ is divergent for $D_{\Gamma}=2$. From Proposition 3.3, $\Gamma^{\prime}$ is also divergent and $D_{\Gamma^{\prime}}=2$. Lemma 4.6 then implies $f$ is absolutely continuous with respect to $\sigma_{y}$ and $\mu_{x}$. Hence by Lemma 5.1, $f$ preserves cross ratio $\sigma_{y^{-}}$-everywhere. By Proposition 3.2, $\Lambda_{\Gamma^{\prime}}=S^{2}$ and since $\sigma_{y}$ is non-zero constant multiple of Lebesgue measure, we can modify $f$ on the Lebesgue measure null subset of $S^{2}$ to a map which is cross ration preserving on $S^{2}$. We denote the new map also by $f$. By Bourdon's theorem [9], $f$ extends into the space as a isometry, i.e. $\mathbb{H}^{3}$ and $\widetilde{M}$ are isometric. Hence the result follows from Theorem 5.4.

Proof. [Proof. Theorems 1.1 part II] Here $f$ embeds $S^{2}$ into $S_{\infty}$. If we suppose $D_{\Gamma}=D_{\Gamma^{\prime}}=2$, then by using same argument as the proof of Theorem 1.2, $f$ extends to a isometric embedding of $\mathbb{H}^{3}$ into $M$ by [9]. Since $f\left(S^{2}\right)$ is a $\Lambda_{\Gamma}$-invariant closed subset of $S_{\infty}$, by Proposition $3.2, f\left(S^{2}\right)=\Lambda_{\Gamma}$. Hence the boundary space of the isometric embedded image of $\mathbb{H}^{3}$ coincides with $\Lambda_{\Gamma}$, therefore the result follows.

Proof. [Proof. Corollary 1.3] This follows from Propositions 3.1, 3.3, and Theorem 1.2. $\square$ 


\section{REFERENCES}

[1] Anderson, M., Schoen, R., Positive harmonic functions on complete manifolds of negative curvature, Ann. Math., 121 (1985), pp. 429-461.

[2] Ancona, A., Negatively curved manifolds, Elliptic operators, and Martin boundary, Ann. Math., 122 (1990), pp. 495-536.

[3] AnconA, A., Théorie du potentiel sur les graphes et les variétés, Lect. Notes in Math., 1427 (1990), Springer, Berlin.

[4] Ballman, W., Gromov, M., Schroeder, V., Manifolds of nonpositive curvature, Birkhäuser, Boston, (1985).

[5] Benedetti, R., Petronio, C., Lecture on hyperbolic geometry, Springer, Universitext, (1992).

[6] Besse, A., Einstein manifolds, Springer, Berlin, (1986).

[7] Besson, G., Courtois, G., Gallot, S., Entropies et rigidités des espaces localement symétriques de courbure strictement négative, G.A.F.A, 5 (1995), pp. 731-799.

[8] Bonahon, F., Bouts des variétés hyperboliques de dimension 3, Ann. Math., 124 (1986), pp. 71-158.

[9] Bourdon, M., Sur le birapport au bord des CAT(-1)-espaces, IHES. Publ. Math, No. 83, (1996).

[10] CanARY, R., Ends of hyperbolic 3-manifolds, J. Amer. Math. Soc., 6 (1993), pp. 1-35.

[11] CoRlette, K., Hausdorff dimension of limit sets I, Invent. Math., 102 (1990), pp. 521-542.

[12] Coornaert, M., Mesures de Patterson-Sullivan, Pacif. J. Math., Vol. 159, No 2, (1990), pp. 241-270.

[13] Douady, A., Earle, C., Conformally nature extension of homeomorphism of the circle, Acta Math., 157 (1986), pp. 23-48.

[14] Feighn, M., Mccullough, D., Finitness conditions for 3-manifolds with boundary, Amer. J. Math., 109 (1987), pp. 1155-1169.

[15] Eberlin, P., O’Neil, B., Visibility manifolds, Pacific. J. Math., 46 (1973), pp. 45-109.

[16] Gabai, D., Meyerhoff, R,. Thurston, N., Homotopy hyperbolic 3-manifolds are hyperbolic MSRI, preprint.

[17] Ebin, D., The manifolds of Riemannian metrics, Global Analysis, editor S.S. Chern and S. Smale. Proc. Symp. Pure. Math., 15 (1970), AMS, Providence.

[18] Fedder, H., Geometric Measure Theory, Springer, 153 (1969).

[19] GaO, L.Z., The construction of negative Ricci curved manifolds, Math. Ann., 271 (1985), pp. 185-208.

[20] Genring, F,W., The definition and exceptional sets for quasiconformal mappings, Ann. Acad. Sci. Fenn. A.I., 281 (1960), pp. 1-28.

[21] GaO, L.Z., YAU, S.T., The existence of negatively Ricci curved metrics on three manifolds, Inv. Math., 85 (1986), pp. 637-652.

[22] Gromov, M., Hyperbolic Groups. In essays in group theory, Gersten (editor) M.S.R.I. Publ., 8 (1987), Springer, Berlin., pp 75-263.

[23] Gromov, M., Schoen, R., Harmonic maps into singular spaces and p-adic superrigidity for lattices in groups of rank-1, Publ. Math. IHES, 78 (1994), pp. 165-246.

[24] Gromov, M., Thurston, W., Pinching constants for hyperbolic manifolds, Inv. Math., 89 (1987), pp. 1-12.

[25] HAMENSTÄDT, U., Entropy-rigidity of locally symmetric spaces of negative curvature, Ann. Math., 131 (1990), pp. 35-51.

[26] HamenstäDt, U., A new description of the Bowen-Margulis measure, Ergod. Th Dynam. Sys., 9 (1989), pp. 455-464.

[27] Heintze, E., Im Hof, H.C., On the geometry of horospheres, J. Diff. Geo., 12 (1977), pp. 481-491.

[28] Hempel, J., 3-manifolds, Ann. Math. Studies., 86 (1976), Princeton Univ. Press.

[29] Hou, Y., Critical exponent and displacement of negatively curved free groups, J. Diff. Geo., vol. 57 (2001), pp. 173-193.

[30] Jost, J., YAU, S. T., Harmonic map and superrigidity, Proc. Symp. Pure. Math. AMS, 54 (1993), pp. 245-280.

[31] KaImanovich, V.A., Invariant Measures for the geodesic flow and measures at infinity on negatively curved manifolds, Ann. Inst. Henri Poincaré, Physique Théorique. 53, No 4 (1990), pp. 361-393.

[32] Margulis, G.A., Discrete subgroups of semisimple Lie groups, Ergebnisse der Math. Springer, NY, (1991).

[33] MEeks, W., YAU, S.T., The equivariant Dehn's lemma and loop theorem, Comm. Math. Helv., 56 (1981), pp. 225-239. 
[34] MoRgan, J., On Thurston's uniformization theorem for thress-dimensional manifolds, The Smith Conjecture. Editor, Morgan. J., Bass. H., Academic Press, New York, pp. 37-125, (1984).

[35] MoK, N., SiU, Y.T., Yeung, S.K., Geometric superrigidity, Invent. Math., 113 (1993), pp. 57-83.

[36] Mostow, G.D., Strong rigidity of locally symmetric spaces, Ann. Math. Studies., 78 (1973), Princeton.

[37] Nicholls, P.J., The ergodic theory of discrete groups, Cambridge Univ. Press., (1989).

[38] Otal, P.J., Thurston's hyperbolization of Haken manifolds, École Normale Supérieure de LYON. Prépublication on Rapport de Recherche $n^{\circ}$ 208, (1997).

[39] Patterson, S.J., Measures on limit sets of Kleinian groups, Analytical and Geometrical Aspects of hyperbolic space. Cambrige Univ. Press., pp 291-323, (1987).

[40] Pansu, P., Dimension conforme et sphere á L'infini des variétés á conrburé négative, Ann. Acad. Sci. Fenn, A 14 (1989), pp. 177-212.

[41] Sullivan, D., Discrete conformal groups and measurable dynamics, Bull. Amer. Math. Soc., 6 (1982), pp. 57-73.

[42] Sullivan, D., The density at infinity of a discrete group of hyperbolic motions, Publ. Math. I.H.E.S., 50 (1979), pp. 171-202.

[43] Sullivan, D., On the ergodic theory at infinity of an arbitrary discrete group of hyperbolic motions, Riemann surface and related topics, Ann. Math. Stud., V97 (1981), pp. 465-496.

[44] Sullivan, D., Discrete conformal groups and measurable dynamics, Bull. AMS, 6 (1982), pp. $57-73$.

[45] Thurston, W., The geometry and topology of 3-manifolds, Lecture notes, Princeton Univ.

[46] YUE, C.B., The ergodic theory of discrete isometry groups on manifolds of variable negative curvature, Trans. Amer. Math. Soc., 348 (1996), pp. 4965-5005.

[47] YuE, C.B., Dimension and Rigidity of Quasi-fuchsian representations, Ann. Math., vol 143, 2 (1996), pp. 331-355. 
